\title{
Pierre-Marc de Biasi, Anne Herschberg Pierrot \& Barbara Vinken, Flaubert. Les pouvoirs du mythe
}

\section{Maria Emanuela Raffi}

\section{(2) OpenEdition}

\section{Journals}

\section{Edizione digitale}

URL: http://journals.openedition.org/studifrancesi/1418

DOI: 10.4000/studifrancesi. 1418

ISSN: 2421-5856

Editore

Rosenberg \& Sellier

\section{Edizione cartacea}

Data di pubblicazione: 1 décembre 2015

Paginazione: 619-620

ISSN: 0039-2944

\section{Notizia bibliografica digitale}

Maria Emanuela Raffi, «Pierre-Marc de Biasi, Anne Herschberg Pierrot \& Barbara Vinken, Flaubert. Les pouvoirs du mythe», Studi Francesi [Online], 177 (LIX | III) | 2015, online dal 01 décembre 2015, consultato il 09 janvier 2021. URL: http://journals.openedition.org/studifrancesi/1418 ; DOI: https:// doi.org/10.4000/studifrancesi. 1418

Questo documento è stato generato automaticamente il 9 janvier 2021.

\section{cc) (i) $\odot$}

Studi Francesi è distribuita con Licenza Creative Commons Attribuzione - Non commerciale - Non opere derivate 4.0 Internazionale. 


\title{
Pierre-Marc de Biasi, Anne Herschberg Pierrot \& Barbara Vinken, Flaubert. Les pouvoirs du mythe
}

\author{
Maria Emanuela Raffi
}

\section{NOTIZIA}

PIERRE-MARC DE BIASI, ANNE HERSCHBERG PIERROT \& BARBARA VINKEN, Flaubert. Les pouvoirs $d u$ mythe, t. 1, Paris, Éditions des archives contemporaines, 2014, pp. 182.

1 La «Renaissance orientale» è lo specifico mito nel segno del quale viene letta, in questa serie di contributi, l'opera di Flaubert, ancora più largamente incorniciata da una riflessione sul mito e la mitografia nei rapporti fra la cultura tedesca e quella francese. L'ampiezza del progetto è chiaramente tracciata da Barbara VINKEN nell'avant-propos del volume e ripresa nell'Introduction di Anne HERSCHBERG PIERROT che, dopo aver mostrato la complessità e ricchezza di contenuti nella combinazione di mito antico e mito moderno in Flaubert, presenta sinteticamente il volume.

2 La prima delle tre parti in cui è diviso il volume affronta «l'intertexte franco-allemand» nel rapporto fra miti e simboli. Michel ESPAGNE tratteggia soprattutto il panorama storico-culturale dello studio del mito sul versante tedesco: da Heyne a Voss e Creuzer e da Welcker a Usener vengono presentati studi filologici di diverso orientamento, ma tutti legati ad una lettura simbolica dei miti antichi. È ciò che accade nella filologia classica tedesca almeno fino all'incontro con gli scritti di Renan nella «Revue germanique» e al nuovo rapporto fra mito e pensiero naturale, che Agnès BouviER illustra nel suo studio Au rendez-vous allemand: la "Revue germanique", ou la philologie allemande au service de la libre pensée française, in cui mette in risalto anche il fondamentale apporto degli articoli scientifici e di quelli relativi all'origine delle lingue 
nel dibattito fra materia e spirito che la rivista ha prodotto. All'importante filologo tedesco Max Müller, introdotto in Francia da Renan, è dedicato l'articolo di Bertrand MARCHAL, Max Müller le tueur des mythes, suivi de Notes sur Flaubert et Müller, in cui l'A. rileva i suoi tre ambiti fondamentali di intervento - «la science du langage», che si occupa del linguaggio non come mezzo ma come oggetto di studio; «la science des mythes», principale settore di investigazione del fondatore della «mythologie comparée» e «la science de la religion» fondata sulla separazione fra mitologia e religione - e la loro influenza su Flaubert. In De Fontenelle à Dupuis: chapitres de l'histoire de la mythographie, Ildikó LÖRINZKY colloca la sua ricerca nel XVIII secolo iniziando da Fontenelle, autore molto apprezzato da Flaubert, e dalla sua idea dell'origine psicologica dei miti, continuando poi con Vico (il mito visto come parte della filosofia della storia) commentato da Michelet e infine con Charles de Brosses, volto a decifrare il carattere feticistico dei miti.

3 La seconda parte del volume - «Idoles. L'empire de l'hagiographie»-considera più da vicino le opere di Flaubert, a partire dallo studio a due voci di Claude MOUCHARD e Jacques NEEFS, Flaubert et l'histoire des religions: "La Tentation de Saint Antoine". Gli autori si interrogano in particolare sull'uso che Flaubert fa dell'enorme erudizione raccolta e delle altrettanto estese «puissances figurales» che essa consente, studiando particolarmente il materiale religioso e il suo incrociarsi con i miti antichi. «La fascination religieuse» è ciò che l'opera flaubertiana mette in scena, sia come percorso nella storia delle religioni, sia come progressione del testo, che passa, nelle diverse edizioni, dal fascino delle divinità all'«extase» della materia e alla potenza fascinatoria della scrittura. In Le défilé des dieux, de l'idole primitive à Loulou, Sylvie TRIAIRE propone una lettura della progressiva soppressione della figura divina («le Dieu d'Israël») nelle successive edizioni della Tentation. Dal legame fra religione e mitologia delle due prime edizioni si passa infatti per l'A. alla separazione della mitologia diventata scienza e oggetto di studio della linguistica negli anni della terza edizione; «le Dieu d'Israël» viene quindi progressivamente abbandonato e separato da un Cristo divenuto simbolo vuoto di «un désir collectif de croyance». Trois contes costituisce per la Triaire la continuazione di quest'opera di separazione di Cristo dal «Dieu d'Israël» e della sua spersonalizzazione. A Hérodias e alla sua particolare versione della nascita del cristianesimo «radicalement différente de la Bible» è dedicato anche lo studio di Barbara VINKEN ("Hérodias". L'Église catholique romaine: fille de Babel) che affronta anzitutto il problema dell'«incompréhensibilité» del racconto, dovuta secondo l'A. alla reinterpretazione dei miti della storia romana che Flaubert opera contro la Chiesa cattolica romana, rovesciando e snaturando le immagini bibliche: «Flaubert situe le christianisme dans le culte de Cybèle et en fait une religion primitive mythique et, en tant que telle, une phallolatrie».

«Mythes. La réécriture symbolique», terza parte del volume, si apre con un contributo di Henri MITTERAND - Zola: le jeu du mythe - che riporta l'attenzione, con la consueta chiarezza e precisione, sull'oggetto letterario, il romanzo, e sul suo funzionamento, la fiction narrativa. Coerentemente, i miti sono considerati come récits e classificati secondo tre tipi fondamentali: «les mythes abstraits» o meglio definiti con nomi astratti che traducono un aspetto dell'esistenza («le paradis perdu», «la fraternité meurtrière», «l'éternel retour», ecc.); «les mythes sans dieux ou noyau thématique originaire», coagulo di senso stratificato su un personaggio reale o fittizio (Don Juan, Robinson Crusoë, Jeanne d'Arc, Schéhérazade, ecc.); «les mythes médiatiques, ou dégradés», 
consacrati da una presenza temporanea nel discorso collettivo ed emblematici di un'epoca (Marilyn Monroe, la DS, ecc.). In letteratura, e in particolare nell'opera di Zola, anche sulla base degli studi di Jean Borie, di Olivier Got e di Roger Ripoll, Mitterand individua il ripresentarsi, da un romanzo all'altro, di una struttura omologabile al mito di Edipo o comunque una struttura mitica di base, benché organizzata in réseaux differenti. Nella grande opera zoliana, accanto ad uno schema sociologico positivista, agisce infatti per Mitterand uno schema mitologico che mette in scena i propri archetipi fondamentali: «le Sexe, la Mort, la Foi et l'Art». In Hippolyte: réinterprétation flaubertienne d'un mythe antique, Edi zOLLINGER si sofferma sulle riprese hugoliane di Flaubert in Madame Bovary e in particolare sui quadri di Steuben di Esmeralda presenti nello studio del notaio Guillaumin/Frollo. Ma il legame più forte viene suggerito per la figura di Hippolyte, ripresa dalle vicende del martire cristiano riprodotte nel quadro di Heim presente nella cattedrale di Notre-Dame e singolarmente somigliante, nella 'tortura' che gli viene inflitta, al personaggio hugoliano di Quasimodo e alle torture minacciate per Esmeralda. Désymbolisation et resymbolisation: per Gisèle SÉGINGER non si tratta di due operazioni incompatibili per Flaubert nel suo rapporto con le religioni. Se la désymbolisation della religione risente anche del dibattito introdotto da Cousin sulla filosofia hegeliana e della diretta lettura dell'Esthétique da parte di Flaubert, l'autore della Tentation e di Salambô arriva tuttavia a conclusioni diverse: «Alors que la désymbolisation implique une pensée du devenir, une dialectique, l'infini flaubertien dépend d'un travail de la forme. Il est du côté du déploiement sans fin de la vie». Pierre-Marc DE BIASI conclude il volume con una riflessione sulla nozione stessa di simbolismo: Le processus symbolique. Symbole et désymbolisation dans "La Légende de Saint Julien l'Hospitalier". Il valore del simbolo per Flaubert appare all'A. soprattutto legato alla sua rarità e alla sorpresa che produce e dunque a generi brevi come l'aforisma e l'apologo, pur nel contesto della vita quotidiana. Il lavoro del lettore flaubertiano appare in buona parte come la ricostruzione di un significato che il processo simbolico del testo presenta come «un manque» in cui inserire «l'autre moitié de l'objet brisé». La «désymbolisation», concetto elaborato negli anni quaranta dell'ottocento e ripreso dalla critica - e dallo stesso De Biasi - a partire dalla fine degli anni 1970, viene qui utilizzato per l'immagine di «Julien tueur de dragon», dove il progressivo modificarsi del nome del mostro evocato in relazione a diverse leggende, lascia trasparire la presenza del «conteur» che si appella alla veridicità della sua versione e lavora a sua volta per la «désymbolisation». 